\title{
8 propuestas para 8 décadas
}

\section{8 proposals for 8 decades}

\author{
José Luis Sandoval-Gutiérrez*
}

\author{
*Instituto Nacional de Enfermedades Respiratorias \\ Ismael Cosío Villegas, Ciudad de México.
}

El Dr. Rendón, ${ }^{1}$ presidente de nuestra sociedad, manda un mensaje sobre los nuevos proyectos de la misma en concordancia con los 80 años de la revista Neumología y Cirugía de Tórax, es importante señalar que es necesario redoblar esfuerzos a través de estas trincheras. Como bien se señala, las enfermedades respiratorias en conjunto son la primera causa de muerte en el mundo ${ }^{2}$ y en México, ${ }^{3}$ aún no se ha dilucidado la importancia de la adecuada enseñanza de la Neumología en las diferentes escuelas y facultades de medicina, se necesitan más especialistas en nuestro país. ${ }^{4,5}$ Todas estas iniciativas se acoplan en medida a lo propuesto por el Foro Internacional de Sociedades Respiratorias (FIRS): ${ }^{6}$

1. Aumentar la conciencia pública y de los encargados de formular políticas de que la salud respiratoria es esencial para la salud global y que la enfermedad respiratoria infantil puede tener consecuencias negativas a largo plazo en la salud de los adultos al abogar en las reuniones mundiales de salud y a través de publicaciones en los medios.

2. Reducir y luego eliminar el uso de todos los productos de tabaco a través del apoyo universal del Convenio Marco para el Control del Tabaco.

3. Adoptar las normas de la OMS, como mínimo, para reducir la contaminación del aire ambiental, interior y ocupacional para todos los países.

4. Promover el acceso universal a una atención médica de calidad, incluida la disponibilidad de medicamentos esenciales, asequibles y de calidad garantizada,

Correspondencia:

Dr. José Luis Sandoval Gutiérrez

Instituto Nacional de Enfermedades Respiratorias

Ismael Cosío Villegas, Ciudad de México

Correo electrónico: sandovalgutierrez@gmail.com y la cobertura universal para las inmunizaciones de niños y adultos, incluidas las nuevas vacunas conjugadas a través de programas de la OMS y del gobierno.

5. Mejorar el diagnóstico precoz de las enfermedades respiratorias a través de la mejora de la conciencia y el acceso a los procedimientos actuales y el desarrollo de nuevas herramientas a través de reuniones y publicaciones mundiales de salud.

6. Aumentar la educación y la formación de profesionales de la salud en enfermedades respiratorias en todo el mundo a través de programas de las sociedades FIRS, la OMS y otras organizaciones gubernamentales y no gubernamentales.

7. Estandarizar el monitoreo de la prevalencia, gravedad y manejo de enfermedades respiratorias para permitir el desarrollo de estrategias nacionales bien informadas a través de programas de la OMS y organizaciones gubernamentales y no gubernamentales.

8. Aumentar la investigación respiratoria para desarrollar programas, herramientas y estrategias para prevenir y tratar mejor las enfermedades respiratorias a través de la promoción de organizaciones de investigación gubernamentales y no gubernamentales.

Con todo lo anterior, lograremos el anhelado deseo de una mejor salud respiratoria para todos en la próxima década.

\section{REFERENCIAS}

1. Rendón PL. Mensaje del Presidente de la Sociedad Mexicana de Neumología y Cirugía de Tórax 2019-2021. Neumol Cir Torax 2019;78(3): 268-269. https://dx.doi.org/10.35366/NT193A.

2. Sandoval GJ, Reyes ES, Bautista EB. Pulmonary diseases: first cause of mortality in the world. Chest 2011;139(6):1550. https://doi. org/10.1378/chest.11-0021. 
Neumol Cir Torax. 2020; 79 (2): 121-122

3. Sandoval GJ. Neumopatías: primera causa de mortalidad en México. Un ejercicio aritmético. Neumol Cir Torax 2013;72(1):121-122.

4. Vázquez GJ, Fernández VM, Salas HJ, Pérez PR. Retos y avances en la formación de especialistas en enfermedades respiratorias en México. Neumol Cir Torax 2012;71(2):147-157.
5. Sandoval GJ. ¿Qué tipo de neumólogos necesitamos? La importancia de la educación médica continua. Neumol Cir Torax 2012;71(2): 198-199.

6. The global impact of respiratory diseases. 2th ed. FIRS. Fecha de recepción: 20 de noviembre, 2019. (https://www.who.int/gard/publications/The_Global_Impact_of_Respiratory_Disease.pdf. 